\title{
Quantum Error-Correction for Spatially Correlated Errors
}

\author{
T. Arimitsu, T. Hayashi* \\ Graduate School of Pure and Applied Sciences, University of Tsukuba \\ Ibaraki 305-8571, Japan \\ tarimtsu@sakura.cc.tsukuba.ac.jp \\ S. Kitajima, F. Shibata \\ Department of Physics, Faculty of Science, Ochanomizu University \\ Tokyo 112-8610, Japan
}

January 31, 2008

\begin{abstract}
It is shown that errors due to spatially correlated noises can be corrected by the quantum error-correction code and error-correction procedure prepared for those for independent noises. A model of noisy-channel which is under the influence of spatially correlated quantum Brownian motion is investigated within the framework of non-equilibrium thermo field dynamics that is a canonical operator formalism for dissipative quantum systems.
\end{abstract}

Dissipative interactions between the system of qubits and its environment produce unexpected transitions of the qubits in a stochastic manner, and cause errors in the quantum information (codeword) stored in the qubits. In order to recover the original information, quantum error-correction codes have been developed $[1,2,3,4,5]$. However, the existing quantum error-correction codes are designed under the hypothesis that each qubit is suffering from noises that are spatially and/or temporally independent $[1,6,7,8,9]$. However, the validity of the hypothesis is not evident in practical circumstances of quantum information processing [1]. The spatial independence of noise is realized only when the distance between adjacent qubits is quite large compared to the correlation length of noise. Therefore, it should be investigated how well the existing quantum error-correction codes, created under the hypothesis, function in such realistic situations where noise is considered to be correlated.

In this paper, we will show that errors due to spatially correlated noises can be corrected by the same quantum error-correction code and error-correction procedure as those for independent noises by investigating a model of noisy-channel which is

*Present address: M.C.C., 1-4-1 Koishikawa, Bunkyo-ku, Tokyo 112-0002, Japan 
under the influence of spatially correlated quantum Brownian motion. This result is valid generally for the stabilizer code [5], which is quite a large class of quantum error-correction codes including the 3-qubit bit-flip code, Shor's 9-qubit code [1], the CSS code [3, 4].

Our analysis is presented in terms of non-equilibrium thermo field dynamics (NETFD) $[10,11,12,13,14]$ that provides us with a full set of methods to tackle problems in quantum information theory, especially, those related to decoherence or dissipation. NETFD is a canonical operator formalism to treat non-equilibrium quantum systems in a similar way as quantum field theory, i.e., the sophisticated interpretations of nature is possible based on both the operator algebra and the representation space. Within NETFD, the dynamics of dissipative quantum systems is described by the "Schrödinger equation" for unstable vacuum, which has the same amount of information contained in the quantum master equation for density operator. In fact, a consistent and unified system of stochastic differential equations in operator formalism, together with a theory of quantum Brownian motion, allows one to analyze time-evolution under correlated noise in a practical manner. In addition, by the introduction of tilde degrees of freedom, completely positive maps (CP maps) describing the error or the error-correction procedure are represented within NETFD by operators acting on both bra- and ket-thermal vacuums for qubits, which makes analyses of error-correction procedures transparent; this makes a contrast to the case within the density operator formalism where $\mathrm{CP}$ maps are super-operator acting on density operator.

The dynamics of the noisy channel, described by the "Schrödinger equation" $\partial|0(t)\rangle / \partial t=\hat{\Pi}|0(t)\rangle$ for the ket-vacuum $|0(t)\rangle$ within NETFD, is solved, with the initial condition $|0(t=0)\rangle=|0\rangle$, to give $|0(t)\rangle=\hat{\mathcal{E}}(t)|0\rangle$ with the spatially correlated error operator [15]

$$
\hat{\mathcal{E}}(t)=\mathrm{e}^{t \hat{\Pi}}, \quad \hat{\Pi}=\frac{1}{2} \sum_{\alpha, \beta \in \mathcal{G}^{(1)}} D_{\alpha \beta}^{*}(2 \alpha \tilde{\beta}-\beta \alpha-\tilde{\alpha} \tilde{\beta}) .
$$

Here, $\alpha$ and $\beta$ stand for an element in $\mathcal{G}^{(1)} \equiv\left\{X_{i}, Y_{i}, Z_{i}\right\}_{i=1}^{n}$ where $X_{i}, Y_{i}$ and $Z_{i}$ represent the Pauli operators acting on the qubit at the $i$ th site. The c-number matrix $D$, the correlation matrix, is an Hermitian matrix with positive eigenvalues characterizing physical nature of noises affecting qubits. Since the noises are supposed to be spatially correlated, $D_{\alpha_{i} \beta_{j}} \neq 0($ for $i \neq j)$ in general, where $\alpha_{i} \in\left\{X_{i}, Y_{i}, Z_{i}\right\}$ and $\beta_{j} \in\left\{X_{j}, Y_{j}, Z_{j}\right\}$. Note that $\hat{\Pi}$ has zero eigenvalue for bra-vacuum $\langle\theta|$, i.e.,

$$
\langle\theta| \hat{\Pi}=0,
$$

which is a manifestation of the conservation of probability, i.e., $\langle\theta \mid O(t)\rangle=1$. Note also that the bra-vacuum has the property

$$
\langle\theta| \tilde{\alpha}=\langle\theta| \alpha^{\dagger}
$$

for $\alpha \in \mathcal{G}^{(1)}$, which is called thermal state condition for the bra-vacuum. 
Because of spatial correlation of noise, $\hat{\mathcal{E}}(t)$ cannot be written as a product of error operators for individual qubit. Note that $\hat{\mathcal{E}}(t)$ is completely positive due to the positivity of the correlation matrix $D$. A block of $n$ qubits can suffer errors like $X_{i}, Z_{i}, Y_{i}, X_{i} X_{j}, X_{i} Y_{j} Z_{k}$, etc.. Thus, it is natural to consider the group $\mathcal{G}=$ $\{ \pm I, \pm X, \pm i Y, \pm Z\}^{\otimes n}$, the Pauli group, which is formed by all possible products of operators $\left\{ \pm I_{i}, \pm X_{i}, \pm i Y_{i}, \pm Z_{i}\right\}_{i=1}^{n}$ for $n$ qubits. The properties of the Pauli group read, for $\forall g, g^{\prime} \in \mathcal{G}$, 1) $g^{2}=I$ or $\left.-I, 2\right) g^{-1}=g^{\dagger}$, and 3) $\left[g, g^{\prime}\right]=0$ or $\left[g, g^{\prime}\right]_{+}=0$.

When the adjacent qubits are well separated, the noise affecting each qubit becomes statistically independent, i.e., $D_{\alpha_{i} \beta_{j}} \propto \delta_{i, j}$. In a more extreme case where the noises acting on each qubits are mutually independent, we have $D_{\alpha \beta}=D_{\alpha} \delta_{\alpha, \beta}$. This "fully independent" correlation matrix leads to

$$
\hat{\mathcal{E}}_{0}(t)=\mathrm{e}^{t \hat{\Pi}_{0}}, \quad \hat{\Pi}_{0}=\sum_{\alpha \in \mathcal{G}^{(1)}} D_{\alpha}(\alpha \tilde{\alpha}-1) .
$$

Note that, within the first order in $t, \hat{\mathcal{E}}_{0}(t)$ gives the depolarizing channel.

For a given subspace $\mathcal{C}(\neq\{0\})$ of the total Hilbert space $\mathcal{H}_{2}^{\otimes n}$, elements $g(\in \mathcal{G})$ that behaves as $I$ on $\mathcal{C}$ form an Abelian subgroup $\mathcal{S}=\{g \in \mathcal{G}|g| \phi\rangle=|\phi\rangle, \forall|\phi\rangle \in \mathcal{C}\}$ which is called the stabilizer of $\mathcal{C}$. Note that $g^{-1}=g^{\dagger}=g$ (i.e., $g^{2}=I$ ) for $\forall g \in \mathcal{S}$. The set of elements $g(\in \mathcal{G})$ commuting with all the elements of the stabilizer $\mathcal{S}$, i.e., $\mathcal{N}_{\mathcal{S}}=\left\{g \in \mathcal{G} \mid\left[g, g^{\prime}\right]=0, \forall g^{\prime} \in \mathcal{S}\right\}$, is called the commutant of the stabilizer. Since $\mathcal{S}$ is Abelian, $\mathcal{S} \subseteq \mathcal{N}_{\mathcal{S}}$. Note that $\left\langle\phi^{\prime}|g| \phi\right\rangle=0$ for $\forall g \in \mathcal{G}-\mathcal{N}_{\mathcal{S}}$ and $\forall|\phi\rangle,\left|\phi^{\prime}\right\rangle \in \mathcal{C}$. This is because, for such a $g\left(\in \mathcal{G}-\mathcal{N}_{\mathcal{S}}\right)$, there exists at least one element $g^{\prime}(\in \mathcal{S})$ satisfying $\left[g, g^{\prime}\right]_{+}=0$, and thus, $\left\langle\phi^{\prime}|g| \phi\right\rangle=\left\langle\phi^{\prime}\left|g^{\prime} g\right| \phi\right\rangle=-\left\langle\phi^{\prime}\left|g g^{\prime}\right| \phi\right\rangle=-\left\langle\phi^{\prime}|g| \phi\right\rangle$ for $\forall|\phi\rangle,\left|\phi^{\prime}\right\rangle \in \mathcal{C}$.

One can say, without loss of generality, that the quantum error-correction codes are constructed for the error operator of the form

$$
\hat{\mathcal{E}}_{0}=\sum_{\mu=0}^{m} p_{\mu} E_{\mu} \tilde{E}_{\mu}, \quad \text { with } E_{\mu} \in \mathcal{G} \text { and } \sum_{\mu=0}^{m} p_{\mu}=1\left(p_{\mu} \geq 0\right) .
$$

The condition for errors created by the error-operator to be corrected on the code $\mathcal{C}$ stabilized by $\mathcal{S}$ reads

$$
E_{\mu}, E_{\mu}^{\dagger} E_{\nu} \in\left(\mathcal{G}-\mathcal{N}_{\mathcal{S}}\right) \cup \mathcal{S} \text { for all } \mu, \nu .
$$

The condition $E_{\mu} \in\left(\mathcal{G}-\mathcal{N}_{\mathcal{S}}\right) \cup \mathcal{S}$ ensures that the error $E_{\mu}$ is detectable or harmless. On the other hand, the condition $E_{\mu}^{\dagger} E_{\nu} \in\left(\mathcal{G}-\mathcal{N}_{\mathcal{S}}\right) \cup \mathcal{S}$ ensures that two errors $E_{\mu}, E_{\nu}$ are distinguishable or equivalent. Note that $E_{\mu}$ transforms the code $\mathcal{C}$ into a subspace $E_{\mu} \mathcal{C}$ of the same dimension, since $E_{\mu}(\in \mathcal{G})$ is unitary. In the following, we arrange $E_{\mu}$ as $E_{0}=I$ and $E_{\mu \geq 1} \neq I$.

The recovery operator $\hat{\mathcal{R}}$ against the error operator (5) with $\left\{E_{\mu}\right\}$ satisfying the error-correction condition (6) is given by

$$
\hat{\mathcal{R}}=\sum_{\mathbf{x} \in \mathbf{Z}_{2}^{s}} g_{\mathbf{x}}^{\dagger} \tilde{g}_{\mathbf{x}}^{\dagger} P_{\mathbf{x}} \tilde{P}_{\mathbf{x}}, \quad P_{\mathbf{x}}=\prod_{a=1}^{s} \frac{1+(-1)^{x_{a}} M_{a}}{2} .
$$


Here, $P_{\mathbf{x}}$ is the projection operator [9], and $\left\{M_{a}\right\}_{a=1}^{s}$ the generator of $\mathcal{S}$ satisfying

$$
E M_{a} E^{\dagger}=(-1)^{x_{a}(E)} M_{a}
$$

with $\mathbf{x}(E) \equiv\left[x_{1}(E), \ldots, x_{s}(E)\right]\left(x_{a}(E) \in \mathbf{Z}_{2}, s=\operatorname{dim} \mathcal{S}\right)$ being the syndrome of an error $E(\in \mathcal{G})$. Here, $\mathbf{Z}_{2} \equiv\{0,1\}$ is the binary field. The element $g_{\mathbf{x}}(\in \mathcal{G})$ appeared in (7) satisfies $\mathbf{x}\left(g_{\mathbf{x}^{\prime}}\right)=\mathbf{x}^{\prime}$, and is determined by the algorithm:

$$
\begin{aligned}
& \text { if } \mathbf{x}^{\prime}=\mathbf{0} \text {, then } g_{\mathbf{x}^{\prime}}=I, \\
& \text { if } \mathbf{x}^{\prime}=\mathbf{x}\left(E_{\mu}\right) \text {, then } g_{\mathbf{x}^{\prime}}=\left(\text { one out of }\left\{E_{\nu} \mid \mathbf{x}\left(E_{\nu}\right)=\mathbf{x}\left(E_{\mu}\right)\right\}\right) \text {. }
\end{aligned}
$$

Here, "one out of" means that any one out of the set is equally eligible for $g_{\mathbf{x}^{\prime}} .{ }^{1}$

$P_{\mathbf{x}}$ is the projection operator onto the simultaneous eigenspace of generators $M_{a}$ with eigenvalues $(-1)^{x_{a}}$. Each factor $\left[1+(-1)^{x_{a}} M_{a}\right] / 2$ on the rhs of the second equation in (7) is the projection operator onto the eigenspace of $M_{a}$ with eigenvalue $(-1)^{x_{a}}$. Thus, the product of them is the projection operator onto the intersection of those eigenspaces. Especially, $P_{\mathbf{0}} \equiv P_{[0, \ldots, 0]}$ is the projection operator onto the code $\mathcal{C}$. $P_{\mathbf{x}}$ 's satisfy the orthogonality condition

$$
P_{\mathbf{x}} P_{\mathbf{x}^{\prime}}=\delta_{\mathbf{x}, \mathbf{x}^{\prime}} P_{\mathbf{x}}
$$

where $\delta_{\mathbf{x}, \mathbf{x}^{\prime}}=\prod_{a=1}^{s} \delta_{x_{a}, x_{a}^{\prime}}$, and the completeness condition

$$
\sum_{\mathbf{x} \in \mathbf{Z}_{2}^{s}} P_{\mathbf{x}}=I
$$

that is consistent with

$$
\langle\theta| \hat{\mathcal{R}}=\langle\theta|
$$

which follows from the thermal state condition (3) and the completeness condition (12). We see that, for $\forall g \in \mathcal{G}$,

$$
g P_{\mathbf{0}} g^{\dagger}=P_{\mathbf{x}(g)} .
$$

Simultaneous measurements of the generators $\left\{M_{a}\right\}_{a=1}^{s}$ on the corrupted codeword $E\left|\phi_{\mathrm{L}}\right\rangle$ tell the syndrome $\mathbf{x}(E)$ via the results $\left\{m_{a}\right\}_{a=1}^{s}$ of the measurements as $m_{a}=(-1)^{x_{a}(E)}$, since $M_{a}\left(E\left|\phi_{\mathrm{L}}\right\rangle\right)=(-1)^{x_{a}(E)} E M_{a}\left|\phi_{\mathrm{L}}\right\rangle=(-1)^{x_{a}(E)}\left(E\left|\phi_{\mathrm{L}}\right\rangle\right)$ for $\forall\left|\phi_{\mathrm{L}}\right\rangle \in \mathcal{C}$. The syndrome $\mathbf{x}(E)$ provides us with necessary and sufficient information to detect and correct the error. By definition, it satisfies the properties: $\mathbf{x}\left(E E^{\prime}\right)=\mathbf{x}(E)+\mathbf{x}\left(E^{\prime}\right)(\bmod 2), \mathbf{x}(E)=\mathbf{x}\left(E^{\dagger}\right)$, and $\mathbf{x}(E)=\mathbf{x}\left(E^{\prime}\right) \Leftrightarrow \mathbf{x}\left(E E^{\prime}\right)=$ $\mathbf{0} \Leftrightarrow E E^{\prime} \in \mathcal{N}_{\mathcal{S}}$. Due to the latter two properties, we have, for the error set $\left\{E_{\mu}\right\}$ satisfying the quantum error-correction condition (6), the error syndrome:

$$
\begin{array}{lll}
\text { when } \mathbf{x}\left(E_{\mu}\right)=\mathbf{x}\left(E_{\nu}\right), & & E_{\mu}^{\dagger} E_{\nu} \in \mathcal{S}, \\
\text { when } \mathbf{x}\left(E_{\mu}\right) \neq \mathbf{x}\left(E_{\nu}\right), & & E_{\mu}^{\dagger} E_{\nu} \in \mathcal{G}-\mathcal{N}_{\mathcal{S}} .
\end{array}
$$

\footnotetext{
${ }^{1}$ When the code is degenerate $[8,9]$, there exist $E_{\mu}, E_{\nu}\left(E_{\mu} \neq E_{\nu}\right)$ satisfying $\mathbf{x}\left(E_{\mu}\right)=\mathbf{x}\left(E_{\nu}\right)$.
} 
Furthermore, with the last property and the quantum error-correction condition (6), we see, for all $E_{\mu}$,

$$
g_{\mathbf{x}\left(E_{\mu}\right)}^{\dagger} E_{\mu} \in \mathcal{S} .
$$

Thus, according to the syndrome, one can apply $E_{\mu}^{-1}\left(=E_{\mu}^{\dagger}\right)$ to retrieve the original codeword $\left|\phi_{\mathrm{L}}\right\rangle$. When two different errors $E_{\mu}$ and $E_{\nu}$ yield the same syndrome, i.e., $\mathbf{x}\left(E_{\mu}\right)=\mathbf{x}\left(E_{\nu}\right)$, an application of $E_{\mu}^{-1}\left(=E_{\mu}^{\dagger}\right)$ correctly recovers the original codeword even if the error actually occurred is $E_{\nu}$. Note that the syndrome $\mathbf{x}\left(E_{\mu}\right)$ do not depend on the codeword but only the error occurred on it. Therefore, the syndrome measurement does not provide us with any information about the codeword itself, which is necessary for quantum error-correction to be successful.

With the above preparations, we can show that the function of the recovery operator $\hat{\mathcal{R}}$ on the code $\mathcal{C}$ is just like $\hat{\mathcal{E}}_{0}^{-1}$, i.e.,

$$
\begin{aligned}
\hat{\mathcal{R}} \hat{\mathcal{E}}_{0} P_{\mathbf{0}} \tilde{P}_{\mathbf{0}} & =\sum_{\mu=0} p_{\mu} \hat{\mathcal{R}} E_{\mu} \tilde{E}_{\mu} P_{\mathbf{0}} \tilde{P}_{\mathbf{0}} \\
& =\sum_{\mu=0} p_{\mu} \hat{\mathcal{R}} P_{\mathbf{x}\left(E_{\mu}\right)} \tilde{P}_{\mathbf{x}\left(E_{\mu}\right)} E_{\mu} \tilde{E}_{\mu} \\
& =\sum_{\mu=0} p_{\mu} g_{\mathbf{x}\left(E_{\mu}\right)}^{\dagger} \tilde{g}_{\mathbf{x}\left(E_{\mu}\right)}^{\dagger} P_{\mathbf{x}\left(E_{\mu}\right)} \tilde{P}_{\mathbf{x}\left(E_{\mu}\right)} E_{\mu} \tilde{E}_{\mu} \\
& =\sum_{\mu=0} p_{\mu} P_{\mathbf{0}} \tilde{P}_{\mathbf{0}} g_{\mathbf{x}\left(E_{\mu}\right)}^{\dagger} E_{\mu} \tilde{g}_{\mathbf{x}\left(E_{\mu}\right)}^{\dagger} \tilde{E}_{\mu} \\
& =\sum_{\mu=0} p_{\mu} P_{\mathbf{0}} \tilde{P}_{\mathbf{0}} \\
& =P_{\mathbf{0}} \tilde{P}_{\mathbf{0}} .
\end{aligned}
$$

Here, (14) has been used at the second and the fourth equality, (11) at the third, and $\sum_{\mu=0} p_{\mu}=1$ at the last equality. At the fifth equality, used are (17) and the property $g=P_{\mathbf{0}}+\sum_{\mathbf{x} \in \mathbf{Z}_{2}^{s} ;(\mathbf{x} \neq \mathbf{0})} c_{\mathbf{x}} P_{\mathbf{x}}\left(c_{\mathbf{x}}=+1\right.$ or -1$)$ satisfied by $\forall g \in \mathcal{S}$. It can be proved, similarly, that the recovery operator $\hat{\mathcal{R}}$ has the property

$$
\hat{\mathcal{R}} E_{\mu} \tilde{E}_{\nu} P_{\mathbf{0}} \tilde{P}_{\mathbf{0}}=\delta_{\mathbf{x}\left(E_{\mu} E_{\nu}\right), \mathbf{0}} P_{\mathbf{0}} \tilde{P}_{\mathbf{0}}, \quad \text { for } E_{\mu}, E_{\nu} \in \mathcal{G} .
$$

Now, let us investigate the function of $\hat{\mathcal{R}}$ on the error operator $\hat{\mathcal{E}}(t)$, caused by spatially correlated noises. Let $\mathcal{C}_{m}$ be a stabilizer code which can correct $\hat{\mathcal{E}}_{0}(t)$ up to $O\left(\left(t \hat{\Pi}_{0}\right)^{m}\right)$ for $m \neq 0$. The quantum error-correction condition

$$
\alpha_{1}, \quad \alpha_{1} \alpha_{2}, \quad \ldots, \quad \alpha_{1} \ldots \alpha_{2 m} \in\left(\mathcal{G}-\mathcal{N}_{\mathcal{S}_{m}}\right) \cup \mathcal{S}_{m} \quad\left(\forall \alpha_{i} \in \mathcal{G}^{(1)}\right)
$$

is satisfied with $\mathcal{S}_{m}$ being the stabilizer for $\mathcal{C}_{m}$. The corresponding recovery operator $\hat{\mathcal{R}}_{m}$ is determined by the algorithm (9) and (10) for $E_{\mu} \in\left\{\alpha, \cdots, \alpha_{1} \cdots \alpha_{m}\right\}$ with $\alpha, \alpha_{i} \in \mathcal{G}^{(1)}$. We observe that $\hat{\Pi}^{m}$ is a linear combination of products of the forms

$$
\alpha_{1} \ldots \alpha_{2 m}, \quad \alpha_{1} \ldots \alpha_{p} \tilde{\alpha}_{p+1} \ldots \tilde{\alpha}_{2 m} \quad(1 \leq p \leq m),
$$


and their tilde-conjugates, with $\alpha_{i} \in \mathcal{G}^{(1)}$ and $\tilde{\alpha}_{i} \in \tilde{\mathcal{G}}^{(1)}$. It is proved that all these products satisfy the operator relation (19). Therefore, we have

$$
\hat{\mathcal{R}}_{m} \hat{\Pi}^{m} P_{\mathbf{0}} \tilde{P}_{\mathbf{0}}=c P_{\mathbf{0}} \tilde{P}_{\mathbf{0}} \quad(\text { for } m \neq 0)
$$

with $c$ being a c-number that is proved to be zero as follows. Multiplying $\langle\theta|$ on both sides of (22), we have

$$
0=\langle\theta| c P_{\mathbf{0}},
$$

where use has been made of (13), (2) and $\langle\theta| \tilde{P}_{\mathbf{0}}=\langle\theta| P_{\mathbf{0}}$. As $\langle\theta| P_{\mathbf{0}} \neq 0$, (23) gives $c=0$. Then, we have

$$
\hat{\mathcal{R}}_{m} \hat{\Pi}^{m} P_{\mathbf{0}} \tilde{P}_{\mathbf{0}}=0 \quad(\text { for } m \neq 0) .
$$

Similar results hold for $\hat{\Pi}^{\ell}(1 \leq \ell<m)$. Thus, we obtain [15]

$$
\hat{\mathcal{R}}_{m} \hat{\mathcal{E}}(t) P_{\mathbf{0}} \tilde{P}_{\mathbf{0}}=P_{\mathbf{0}} \tilde{P}_{\mathbf{0}}
$$

up to $O\left((t \hat{\Pi})^{m}\right)$ for a positive integer $m$.

Within the present model of spatially correlated noise, we have confirmed through the result (25) that the spatially correlated errors happening on quantum codeword are something which can be dealt with, at each order in $\hat{\Pi}$, by the existing stabilizer code for independent errors, or something which does not contribute to the final state after the correction procedure. This is the reason why spatially correlated errors can be corrected by the same stabilizer code and recovery operation as those for independent errors.

\section{References}

[1] P. W. Shor, Phys. Rev. A 52 (1995) R2493.

[2] A. M. Steane, Phys. Rev. Lett. 77 (1996) 793.

[3] A. R. Calderbank and P. W. Shor, Phys. Rev. A 54 (1996) 1098.

[4] A. Steane, Proc. R. Soc. Lond. A 452 (1996) 2551.

[5] D. Gottesman, Phys. Rev. A 54 (1996) 1862.

[6] J. Preskill, Proc. R. Soc. Lond. A454 (1998) 385.

[7] D. Gottesman, Stabilizer codes and quantum error-correction, quant-ph/9705052.

[8] J. Gruska, Quantum Computing (McGraw-Hill, Inc., 1999).

[9] M. A. Nielsen and I. L. Chuang, Quantum Computation and Quantum Information (Cambridge University Press, 2000).

[10] T. Arimitsu and H. Umezawa, Prog. Theor. Phys. 74 (1985) 429.

[11] T. Arimitsu and H. Umezawa, Prog. Theor. Phys. 77 (1987) 32.

[12] T. Arimitsu and H. Umezawa, Prog. Theor. Phys. 77 (1987) 53.

[13] T. Arimitsu, Phys. Lett. A 153 (1991) 163.

[14] T. Arimitsu, Cond. Matt. Phys. Issue 4 (1994) 26.

[15] T. Hayashi, T. Arimitsu, S. Kitajima and F. Shibata, J. Phys. A: Math. Theor. 40 (2007) F457. 